\title{
A four year audit of deliveries by caeserean section at a medical college hospital in Central India
}

\author{
Malini Bharadwaj, Jyoti Nath Modi*
}

Department of Obstetrics \& Gynaecology, People's College of Medical Sciences \& Research Centre, Bhopal, India

Received: 18 September 2015

Accepted: 03 October 2015

\author{
*Correspondence: \\ Dr. Jyoti Nath Modi, \\ E-mail: modijn@gmail.com
}

Copyright: (C) the author(s), publisher and licensee Medip Academy. This is an open-access article distributed under the terms of the Creative Commons Attribution Non-Commercial License, which permits unrestricted non-commercial use, distribution, and reproduction in any medium, provided the original work is properly cited.

\begin{abstract}
Background: There is a global concern for rising rates of deliveries by Caesarean Section over the past few decades. Caesarean deliveries are not only associated with a higher morbidity and mortality but also a longer duration of hospital stay and greater financial burden. Hence they are justified only where better perinatal outcome is expected. An audit of indications of caesarean sections may help identify the areas of intervention for reducing the caesarean delivery rates.

Methods: The delivery records were analyzed retrospectively from January 2008 to December 2011. The rates and indications for deliveries by caesarean section were analyzed.

Results: The total number of deliveries in the three year period was 4084. Of these, 1965 deliveries were caesarean deliveries $(48.1 \%)$. Year wise, a rising trend in caesarean section rate was noted: $40.8 \%$ in $2008 ; 46 \%$ in $2009 ; 48.7 \%$ in 2010 and $56.5 \%$ in 2011. The leading indication for caesarean section was fetal distress (35\%). The others major indications were previous caesarean delivery (26\%), Cephalo Pelvic Disproportion (10.4\%), Malposition/malpresentation (8.2\%), prolonged labour (7.8\%), Hypertensive disorders of Pregnancy (2.2), Antepartum haemorrhage (2\%) and Obstructed labour (1.7\%). The proportion of CS done for previous caesarean section steadily increased over the four years and a falling trend was noticed for CS done for prolonged labour.

Conclusions: The rate of caesarean section needs to be closely monitored and audited so as to take measures for reducing the caesarean rates. An in depth analysis of caesareans section done for fetal distress and previous CS is recommended so that areas of intervention can be identified. The decision for primary CS should be done after a comprehensive assessment and with due justification.
\end{abstract}

Keywords: Caesarean delivery, Caeserian section rate, Indications

\section{INTRODUCTION}

The concern for rising rate of deliveries by Caesarean Section (CS) has been globally voiced over the past few decades. $^{1-5}$ In 1985, the World Health Organization (WHO) recommended that ideal caesarean rates be between 10 and $15 \%$. This was based on the meeting of a panel of Reproductive health experts in Fortaleza, Brazil. ${ }^{6,7}$ This remained the accepted norm until in 2014, a systematic review and population based study carried out by the WHO concluded that $10-15 \%$ caesarean section rates at population level are associated with decreases in maternal, neonatal and infants mortality. Further, there appears to be no additional benefit at rates above $10 \%$ and up to $30 \%$. $^{8}$ However, at the facility level (hospital level), the WHO recommended that it is essential to monitor the rates of the CS taking into account the specific characteristics of the population that they serve. ${ }^{8}$

Very high rates of CS have been reported from institutions in Brazil (over $80 \%$ in some centres and 
national CS rate of $47.7 \%$ ) and China (national CS rate about $40.5 \%) .{ }^{1,9}$ Though the overall CS rate in Africa in very low (about $3.5 \%$ ) largely due to underutilization of health facilities, some institutions in South Africa report a CS rate of up to $60 \% .{ }^{10}$ Southeast Asian region in particular has seen an unacceptable rise in CS rates. ${ }^{11,12} \mathrm{~A}$ study by Stanton in 2006 reported on annual rate of change of CS rates and found India to have one of highest rates of rise in CS deliveries. ${ }^{3}$ A landmark study by a team from Indian Council of Medical Research (ICMR) in 2002 studied the CS rates at 30 teaching hospitals in India and reported a rise in overall CS rate from $21.8 \%$ (1993-94) to $25.4 \%$ (1998-99). ${ }^{13}$ A paper by Ghosh S, compared the data on deliveries from the three National Family Health Surveys (NFHS) in India and found a consistent rise in overall national CS rates: $2.9 \%$ (NFHS1. 1992-93), $7.1 \%$ (NFHS-2, 1998-99) and $10.2 \%$ (NFHS-3, 2005-2006). ${ }^{14}$ The NFHS-3 (2005-2006) found the overall national institutional delivery rate to be $44.8 \%$ of which $23.7 \%$ women were delivered by CS. The rate is higher in the private health facilities compared to the public health facilities (29.9\% and $18.1 \%$ respectively). ${ }^{14}$

Delivery by caesarean section not only increases the risk of maternal mortality but also of maternal morbidity and influences future deliveries. Maternal problems include wound site pain, prolonged hospital stay, more use of antibiotics and medicines, need for further surgery, possibility of hysterectomy, etc. Additionally, there are social and emotional implications associated such as poor birth experience, late contact with the baby, etc. Several neonatal morbidities such as respiratory problems, accidental surgical cuts, and non-establishment of breast feeding can also occur. Caesarean deliveries require more human resources and also pose a higher financial burden. ${ }^{2,15}$ Auditing of CS rates and indications has been suggested as a tool for limiting the unnecessary caesareans as was well reported by a group from Sri Lanka. $^{16}$

The present paper intends to report the trend in rate of deliveries by Caesarean Section over a four-year period at our institution (teaching hospital in central India) and present an analysis of the indications for the caesarean sections. The overall goal was to identify the areas where we can plan effective interventions to control the rise in CS.

\section{METHODS}

This is a retrospective descriptive analytical study carried out in the department of Obstetrics \& Gynaecology, People's College of Medical Sciences \& Research Centre, Bhopal from Jan 2008 to Dec 2011. The delivery records of this four year period were analyzed to study the changing trend in rate of Caesarean deliveries and the various indications for CS. Subgroup analysis of indications for CS among the multigravidae with previous vaginal delivery was also done. Descriptive statistics was used for analysis and Chi square test was used for comparison of groups. $\mathrm{P}$ value was taken significant at $<0.05$.

\section{RESULTS}

The total number of deliveries in the years 2008, 2009, 2010 and 2011 were 4084, of which 1965 were Caesarean deliveries (48.1\%). There were 41\% (1965/4084) primigravidae (Figure 1). The year wise analysis of Caesarean section rate in the four years is presented in Table 1. The mean age of the patients was 25.7 years [Range 17-45 years; SD 4.4]. Almost $75 \%$ of patients were in the age range 20-29 years and $22 \%$ were in the age range $30-39$ years. Almost $70 \%$ of all patients who had caesarean delivery had received antenatal care and were booked patients of our Antenatal clinic.

In 2008 , the caesarean delivery rate was $40.9 \%$ that rose to $46 \%$ in $2009,48.72 \%$ in 2010 and touched a high rate of $56.5 \%$ in 2011 (Table 1). This constitutes a rise by $38 \%$ from baseline over four years or an annual rate of rise of $9.5 \%$. In all 4 years and also overall, the caesarean rate was significantly higher amongst the primigravidae $(61.8 \%)$ compared to the multigravidae (38.6\%); [Chi square: $4.889 ; \mathrm{p}=0.027]$. The caesarean rate showed a rising trend over the four years in both primigravidae as well as multigravidae (Figure 2). The CS rate rose from $55.6 \%$ to $73.4 \%$ over four years among the primigravidae (32\% rises over baseline or annual rate of rise of $8 \%$ ). The CS rate among the multigravidae also rose from $30.9 \%$ to $46 \%$ over four years (49\% rises over baseline or annual rate of rise of $12.3 \%$ ). Majority $(97.1 \%)$ of patients were operated in emergency, while $2.9 \%$ patients were taken for elective CS (Table 1).

The indications of caesarean section are summarized in Table 2. The most frequent indication for CS over all four years was fetal distress $(34.9 \%)$ followed by prior CS delivery (25.9\%), Cephalo-Pelvic Disproportion (CPD), malposition/malpresentation and prolonged labour (Figure 3). This pattern was observed to be similar in each of the four years individually as well (Figure 4). The frequency of CS done for previous CS showed a rising trend $(23.8 \%$ to $30.6 \%)$ over the four years while the CS for prolonged labour showed a downward trend. All other indications were observed with a similar frequency over the four years (Table 2).

The indications for CS were analysed in the subgroups of women who were primigravida (1036 women) and multigravida (929 women). The comparative analysis of indications in these two subgroups is shown in Table 3. The most frequent indication among the primigravida was fetal distress $(47.5 \%)$ followed by CPD (17.6\%) and prolonged labour (11.6\%). However, among the multigravidae, the most frequent indication was 'previous CS' $^{\prime}(54.8 \%)$ followed by fetal distress (20.8\%). Fetal distress and CPD were significantly less frequent among the multigravidae than the primigravidae. Though 'prolonged labour' as indication for CS was also lower 
among the multigravidae than among the primigravidae, this difference was not statistically significant. The frequency of all other indications was comparable between the two groups.

A further analysis was carried out for indications for CS among multigravida who had no previous CS and had one of more vaginal deliveries (Table 4). There were 420 such women. Fetal distress was the commonest indication accounting for $52 \%$ of $\mathrm{CS}$, which was similar to the primigravidae. This was followed by CS done for malpositions/malpresentations $(14 \%)$ and for prolonged labour $(8.1 \%)$.

Table 1: Rate of delivery by caesarean section in the years 2008 to 2011 ( $N=4084)$.

\begin{tabular}{|c|c|c|c|c|c|}
\hline & $\begin{array}{l}2008 \\
\text { Total deliveries } \\
n=989 \\
(\%)\end{array}$ & $\begin{array}{l}2009 \\
\text { Total deliveries } \\
\mathrm{n}=1027 \\
(\%)\end{array}$ & $\begin{array}{l}2010 \\
\text { Total deliveries } \\
\mathrm{n}=1020 \\
(\%)\end{array}$ & $\begin{array}{l}2011 \\
\text { Total deliveries } \\
\mathrm{n}=1048 \\
(\%)\end{array}$ & $\begin{array}{l}\text { 2008-2011 } \\
\text { Total deliveries } \\
\mathrm{N}=4084 \\
(\%)\end{array}$ \\
\hline $\begin{array}{l}\text { Deliveries by } \\
\text { Caesarean } \\
\text { Section }(\mathrm{CS})\end{array}$ & $\begin{array}{l}404 \\
(40.9)\end{array}$ & $\begin{array}{l}472 \\
(46)\end{array}$ & $\begin{array}{l}497 \\
(48.7)\end{array}$ & $\begin{array}{l}592 \\
(56.5)\end{array}$ & $\begin{array}{l}1965 \\
(48.1)\end{array}$ \\
\hline $\begin{array}{l}\text { CS rate in } \\
\text { Primigravidae* }\end{array}$ & $\begin{array}{l}221 / 397 \\
(55.7)\end{array}$ & $\begin{array}{l}253 / 423 \\
(59.8)\end{array}$ & $\begin{array}{l}267 / 454 \\
(58.8)\end{array}$ & $\begin{array}{l}295 / 402 \\
(73.4)\end{array}$ & $\begin{array}{l}1036 / 1676 \\
(61.8)\end{array}$ \\
\hline $\begin{array}{l}\text { CS rate in } \\
\text { Multigravidae* }\end{array}$ & $\begin{array}{l}183 / 592 \\
(30.9)\end{array}$ & $\begin{array}{l}219 / 604 \\
(36.3)\end{array}$ & $\begin{array}{l}230 / 566 \\
(40.6)\end{array}$ & $\begin{array}{l}297 / 646 \\
(46)\end{array}$ & $\begin{array}{l}929 / / 2408 \\
(38.6)\end{array}$ \\
\hline $\begin{array}{l}\text { Booked } \\
\text { (Received } \\
\text { ANC) }\end{array}$ & $\begin{array}{l}256 / 404 \\
(63.4)\end{array}$ & $\begin{array}{l}421 / 472 \\
(89.2)\end{array}$ & $\begin{array}{l}327 / 497 \\
(65.8)\end{array}$ & $\begin{array}{l}377 / 592 \\
(63.7)\end{array}$ & $\begin{array}{l}1381 / 1965 \\
(70.3 \%)\end{array}$ \\
\hline $\begin{array}{l}\text { Elective } \\
\text { sections }\end{array}$ & $\begin{array}{l}11 \\
(2.7)\end{array}$ & $\begin{array}{l}21 \\
(4.4)\end{array}$ & $\begin{array}{l}11 \\
(2.2)\end{array}$ & $\begin{array}{l}13 \\
(2.2)\end{array}$ & $\begin{array}{l}56 \\
(2.9)\end{array}$ \\
\hline
\end{tabular}

*Difference in CS rate between Primigravidae and Multigravidae: Chi $s q=4.889 ; p=0.027$

Table 2: Indications for caesarean sections in four year period $(2008-2011 ; \mathrm{N}=1965)$.

\begin{tabular}{|c|c|c|c|c|c|}
\hline Indication & $\begin{array}{l}2008 \\
n=404 \\
(\%)\end{array}$ & $\begin{array}{l}2009 \\
\mathrm{n}=472 \\
(\%)\end{array}$ & $\begin{array}{l}2010 \\
n=497 \\
(\%)\end{array}$ & $\begin{array}{l}2011 \\
\mathrm{n}=592 \\
(\%)\end{array}$ & $\begin{array}{l}2008-2011 \\
\mathrm{~N}=1965 \\
(\%)\end{array}$ \\
\hline Fetal distress & $\begin{array}{l}134 \\
(33.1)\end{array}$ & $\begin{array}{l}181 \\
(38.4)\end{array}$ & $\begin{array}{l}186 \\
(37.4)\end{array}$ & $\begin{array}{l}184 \\
(31)\end{array}$ & $\begin{array}{l}686 \\
(34.9)\end{array}$ \\
\hline Previous CS & $\begin{array}{l}96 \\
(23.8)\end{array}$ & $\begin{array}{l}100 \\
(21.2)\end{array}$ & $\begin{array}{l}132 \\
(26.6)\end{array}$ & $\begin{array}{l}181 \\
(30.6)\end{array}$ & $\begin{array}{l}509 \\
(25.9) \\
\end{array}$ \\
\hline Cephalo-Pelvic Disproportion (CPD) & $\begin{array}{l}43 \\
(10.6)\end{array}$ & $\begin{array}{l}58 \\
(12.3) \\
\end{array}$ & $\begin{array}{l}40 \\
(8) \\
\end{array}$ & $\begin{array}{l}64 \\
(10.8)\end{array}$ & $\begin{array}{l}205 \\
(10.4)\end{array}$ \\
\hline Malposition/ Malpresentation & $\begin{array}{l}34 \\
(8.4)\end{array}$ & $\begin{array}{l}42 \\
(8.9)\end{array}$ & $\begin{array}{l}39 \\
(7.9)\end{array}$ & $\begin{array}{l}46 \\
(7.8)\end{array}$ & $\begin{array}{l}161 \\
(8.2)\end{array}$ \\
\hline Prolonged Labour & $\begin{array}{l}46 \\
(11.4) \\
\end{array}$ & $\begin{array}{l}33 \\
(7)\end{array}$ & $\begin{array}{l}34 \\
(6.8)\end{array}$ & $\begin{array}{l}41 \\
(6.9)\end{array}$ & $\begin{array}{l}154 \\
(7.8)\end{array}$ \\
\hline Hypertensive Disorders of Pregnancy (HDP) & $\begin{array}{l}8 \\
(2) \\
\end{array}$ & $\begin{array}{l}7 \\
(1.5)\end{array}$ & $\begin{array}{l}11 \\
(2.2)\end{array}$ & $\begin{array}{l}18 \\
(3)\end{array}$ & $\begin{array}{l}44 \\
(2.2)\end{array}$ \\
\hline Antepartum Haemorrhage (APH) & $\begin{array}{l}8 \\
(2) \\
\end{array}$ & $\begin{array}{l}12 \\
(2.5)\end{array}$ & $\begin{array}{l}7 \\
(1.4)\end{array}$ & $\begin{array}{l}12 \\
(2)\end{array}$ & $\begin{array}{l}39 \\
(2)\end{array}$ \\
\hline Obstructed Labour & $\begin{array}{l}7 \\
(1.7)\end{array}$ & $\begin{array}{l}8 \\
(1.7)\end{array}$ & $\begin{array}{l}7 \\
(1.4)\end{array}$ & $\begin{array}{l}12 \\
(2)\end{array}$ & $\begin{array}{l}34 \\
(1.7)\end{array}$ \\
\hline Bad Obstetric History (BOH) & $\begin{array}{l}10 \\
(2.5)\end{array}$ & $\begin{array}{l}18 \\
(3.8)\end{array}$ & $\begin{array}{l}6 \\
(1.2)\end{array}$ & $\begin{array}{l}2 \\
(0.3)\end{array}$ & $\begin{array}{l}36 \\
(1.8)\end{array}$ \\
\hline Others & $\begin{array}{l}18 \\
(4.4)\end{array}$ & $\begin{array}{l}13 \\
(2.7)\end{array}$ & $\begin{array}{l}35 \\
(7)\end{array}$ & $\begin{array}{l}32 \\
(5.4)\end{array}$ & $\begin{array}{l}98 \\
(5)\end{array}$ \\
\hline
\end{tabular}




\section{DISCUSSION}

The overall rate of CS over four years (2008-2011) at our institution was $48.1 \%$ which is higher than the national institutional CS rate reported in the NFHS-3 in 2005-06 $(23.7 \%) .{ }^{14}$ In this report, for Madhya Pradesh (MP), the institutional delivery rate was $38.3 \%(20.8 \%$ in public health facilities and $17.5 \%$ in private health facilities). The overall caesarean rate for the state of MP was $17.7 \%$ (9.7\% in public and $27.1 \%$ in private health facilities respectively). ${ }^{14}$ However, this national survey was in 2005-06, and going by the trend, the rates are bound to be higher at present. Some tertiary care and teaching hospitals have reported higher CS rates than ours. A five year audit of CS from a large teaching hospital in Kolkata reported a CS rate of $49.9 \%$ in $1997 .{ }^{17}$ Another study from Chennai reported CS rate of $50 \%$ in $2003 .{ }^{18}$ More recently, an ICMR task force study published in 2014, reported the CS rate from 30 teaching hospitals to be $28.1 \%$ in the years $2005-06 .{ }^{19}$ The range of CS rate included a low of $11.6 \%$ at LLR Medical college Meerut and $14.6 \%$ at BJ Medical College Pune to a high of $58.7 \%$ at MLN Medical college Allahabad and 55.6\% at SSKM Hospital, Kolkata. ${ }^{19}$ This was a rise of $2.7 \%$ over the earlier reported rate $(25.4 \%)$ from the same 30 hospitals in 1998-99 by Kambo et al from ICMR. ${ }^{13}$

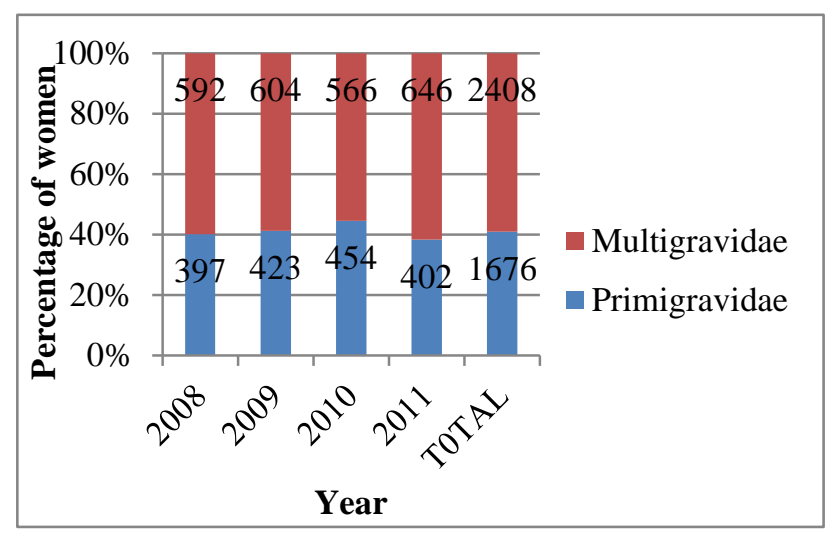

Figure 1: Study group composition: Primigravidae and Multigravidae.

In our study, a rise in CS rate over four years was observed for the whole group (9.5\% annually) as well as among the primigravidae (8\% annually) and multigravidae (12.3\% annually) subgroups. Studies from institutions in Pondicherry and Thiruvananthapuram have reported the CS rates to be $32.6 \%$ (2011) and 40\% (20102011) respectively. ${ }^{20,21}$

Overall, there were $41 \%$ primigravidae in the present study with nearly $75 \%$ patients in the age group 20-29 years. The study by Unnikrishnan et al also reported on a similar group with $47 \%$ women being primigravidae and nearly $82 \%$ women in the age group $21-30$ years. They too reported a majority (87\%) of CS being done in booked ANC patients similar to our findings with a majority of CS in the booked ANC patients (70\%). Study by Ado and Geidem also reported $78.95 \%$ CS being done in booked antenatal cases. ${ }^{4}$

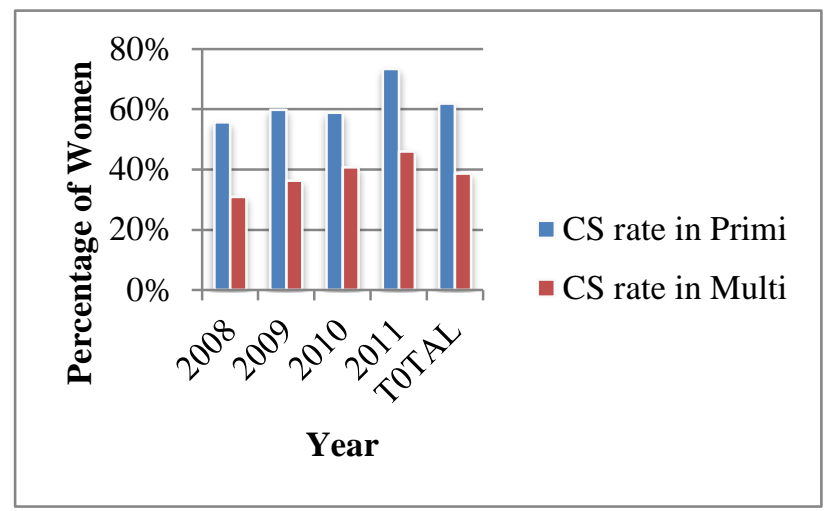

Figure 2: Caesarean section rate among Primigravidae and Multigravidae.

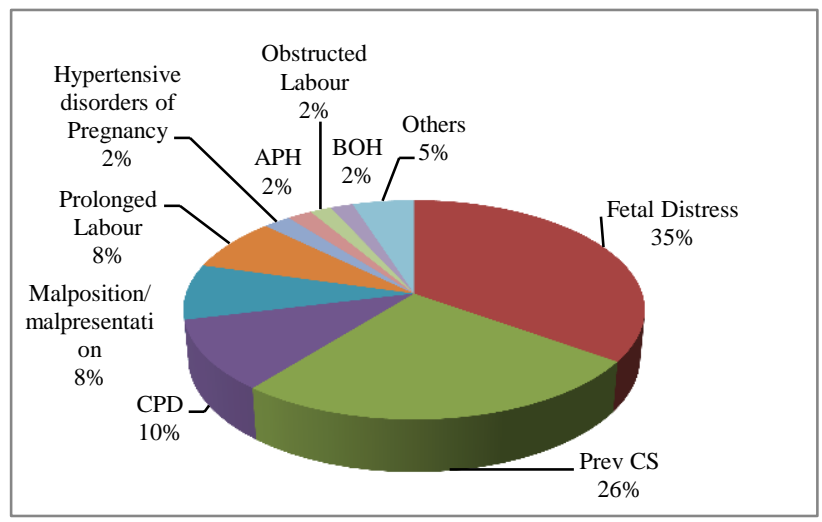

Figure 3: Indications for Caesarean section over all four years: 2008-2011 ( $\mathrm{N}=1965)$.

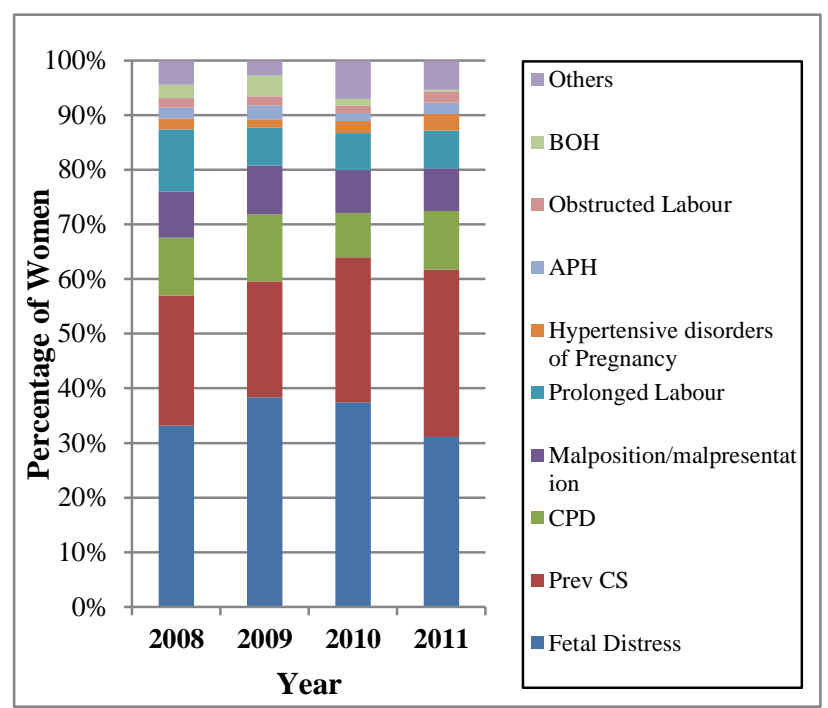

Figure 4: Indications (year wise) for Caesarean section from $2008-2011$.

The CS rate among the primigravidae in our study was significantly higher than among the multigravidae $(61.8 \%$ 
and $38.6 \%$ respectively). The study by Balakrishnan and Nair also reports a CS rate of 50\% among the induced primigravidae and $29 \%$ among the induced multigravidae. $^{21}$ The paper by Roy Choudhary also reports that the CS rates fall with a rise in birth order or increasing parity. ${ }^{2}$ The women with three or more birth order are 0.55 times less likely to have a CS than the primigravidae. $^{2}$ The multivariate analysis in this paper further reports that the chances of CS are higher in urban areas, in private institutions and the mothers who have received Antenatal Care (ANC). ${ }^{2}$ The association with ANC is possibly due to the fact that women with complications are more likely to follow up in ANC; and also the complications are likely to be detected timely among women who have regular ANC visits.

Table 3: Indications for caesarean section among the primigravidae and multigravidae over four years (2008-2011).

\begin{tabular}{|c|c|c|c|c|c|c|c|c|c|c|}
\hline \multirow[b]{2}{*}{ Indications } & \multicolumn{5}{|c|}{ Primigravidae } & \multicolumn{5}{|c|}{ Multigravidae } \\
\hline & 2008 & 2009 & 2010 & 2011 & $\begin{array}{l}\text { All four } \\
\text { years } \\
(2008- \\
2011)\end{array}$ & 2008 & 2009 & 2010 & 2011 & $\begin{array}{l}\text { All } \\
\text { four } \\
\text { years } \\
(2008- \\
2011)\end{array}$ \\
\hline $\begin{array}{l}\text { Caesarean } \\
\text { Deliveries in } \\
\text { Primigravidae }\end{array}$ & $\begin{array}{l}\mathrm{n}=221 \\
(\%)\end{array}$ & $\begin{array}{l}\mathrm{n}=253 \\
(\%)\end{array}$ & $\begin{array}{l}\mathrm{n}=267 \\
(\%)\end{array}$ & $\begin{array}{l}\mathrm{n}=295 \\
(\%)\end{array}$ & $\begin{array}{l}\mathrm{N}=1036 \\
(\%)\end{array}$ & $\begin{array}{l}\mathrm{n}=183 \\
(\%)\end{array}$ & $\begin{array}{l}\mathrm{n}=219 \\
(\%)\end{array}$ & $\begin{array}{l}\mathrm{n}=230 \\
(\%)\end{array}$ & $\begin{array}{l}\mathrm{n}=297 \\
(\%)\end{array}$ & $\begin{array}{l}\mathrm{N}=929 \\
(\%)\end{array}$ \\
\hline Fetal Distress* & $\begin{array}{l}99 \\
(44.8)\end{array}$ & $\begin{array}{l}123 \\
(48.6)\end{array}$ & $\begin{array}{l}141 \\
(52.8)\end{array}$ & $\begin{array}{l}129 \\
(43.7)\end{array}$ & $\begin{array}{l}492 \\
(47.5)\end{array}$ & $\begin{array}{l}35 \\
(19.1)\end{array}$ & $\begin{array}{l}58 \\
(26.5)\end{array}$ & $\begin{array}{l}45 \\
(19.6)\end{array}$ & $\begin{array}{l}55 \\
(18.5)\end{array}$ & $\begin{array}{l}193 \\
(20.8)\end{array}$ \\
\hline Previous CS & - & - & - & - & - & $\begin{array}{l}96 \\
(52.5)\end{array}$ & $\begin{array}{l}100 \\
(45.7) \\
\end{array}$ & $\begin{array}{l}132 \\
(57.4)\end{array}$ & $\begin{array}{l}181 \\
(60.9)\end{array}$ & $\begin{array}{l}509 \\
(54.8)\end{array}$ \\
\hline $\begin{array}{l}\text { Cephalo-Pelvic } \\
\text { Disproportion } \\
\text { (CPD) }\end{array}$ & $\begin{array}{l}40 \\
(18.1)\end{array}$ & $\begin{array}{l}51 \\
(20.2)\end{array}$ & $\begin{array}{l}34 \\
(12.7)\end{array}$ & $\begin{array}{l}57 \\
(19.3)\end{array}$ & $\begin{array}{l}182 \\
(17.6)\end{array}$ & $\begin{array}{l}3 \\
(1.6)\end{array}$ & $\begin{array}{l}7 \\
(3.2)\end{array}$ & $\begin{array}{l}6 \\
(2.6)\end{array}$ & $\begin{array}{l}7 \\
(2.4)\end{array}$ & $\begin{array}{l}23 \\
(2.5)\end{array}$ \\
\hline $\begin{array}{l}\text { Prolonged } \\
\text { Labour } \$\end{array}$ & $\begin{array}{l}35 \\
(15.8)\end{array}$ & $\begin{array}{l}27 \\
(10.7)\end{array}$ & $\begin{array}{l}30 \\
(11.20\end{array}$ & $\begin{array}{l}28 \\
(9.5)\end{array}$ & $\begin{array}{l}120 \\
(11.6)\end{array}$ & $\begin{array}{l}11 \\
(6)\end{array}$ & $\begin{array}{l}6 \\
(2.7)\end{array}$ & $\begin{array}{l}4 \\
(1.7)\end{array}$ & $\begin{array}{l}13 \\
(4.4)\end{array}$ & $\begin{array}{l}34 \\
(3.7)\end{array}$ \\
\hline $\begin{array}{l}\text { Malposition/ } \\
\text { Malpresentation }\end{array}$ & $\begin{array}{l}20 \\
(9.1)\end{array}$ & $\begin{array}{l}26 \\
(10.3)\end{array}$ & $\begin{array}{l}24 \\
(9)\end{array}$ & $\begin{array}{l}34 \\
(11.5)\end{array}$ & $\begin{array}{l}104 \\
(10)\end{array}$ & $\begin{array}{l}14 \\
(7.7)\end{array}$ & $\begin{array}{l}16 \\
(7.3)\end{array}$ & $\begin{array}{l}15 \\
(6.5)\end{array}$ & $\begin{array}{l}12 \\
(4)\end{array}$ & $\begin{array}{l}57 \\
(6.1)\end{array}$ \\
\hline $\begin{array}{l}\text { Hypertensive } \\
\text { Disorders of } \\
\text { Pregnancy } \\
\text { (HDP) }\end{array}$ & $\begin{array}{l}7 \\
(3.2)\end{array}$ & $\begin{array}{l}6 \\
(2.4)\end{array}$ & $\begin{array}{l}6 \\
(2.3)\end{array}$ & $\begin{array}{l}8 \\
(2.7)\end{array}$ & $\begin{array}{l}27 \\
(2.6)\end{array}$ & $\begin{array}{l}1 \\
(0.5)\end{array}$ & $\begin{array}{l}1 \\
(0.5)\end{array}$ & $\begin{array}{l}5 \\
(2.2)\end{array}$ & $\begin{array}{l}10 \\
(3.4)\end{array}$ & $\begin{array}{l}17 \\
(1.8)\end{array}$ \\
\hline $\begin{array}{l}\text { Obstructed } \\
\text { Labour }\end{array}$ & $\begin{array}{l}6 \\
(2.7)\end{array}$ & $\begin{array}{l}5 \\
(2)\end{array}$ & $\begin{array}{l}3 \\
(1.1)\end{array}$ & $\begin{array}{l}8 \\
(2.7)\end{array}$ & $\begin{array}{l}22 \\
(1.9)\end{array}$ & $\begin{array}{l}1 \\
(0.5)\end{array}$ & $\begin{array}{l}3 \\
(1.4)\end{array}$ & $\begin{array}{l}4 \\
(1.7)\end{array}$ & $\begin{array}{l}4 \\
(1.3)\end{array}$ & $\begin{array}{l}12 \\
(1.3)\end{array}$ \\
\hline $\begin{array}{l}\text { Antepartum } \\
\text { Haemorrhage } \\
\text { (APH) }\end{array}$ & $\begin{array}{l}3 \\
(1.4)\end{array}$ & $\begin{array}{l}5 \\
(2)\end{array}$ & $\begin{array}{l}4 \\
(1.5)\end{array}$ & $\begin{array}{l}6 \\
(2)\end{array}$ & $\begin{array}{l}18 \\
(1.7)\end{array}$ & $\begin{array}{l}5 \\
(2.7)\end{array}$ & $\begin{array}{l}7 \\
(3.2)\end{array}$ & $\begin{array}{l}3 \\
(1.3)\end{array}$ & $\begin{array}{l}6 \\
(2)\end{array}$ & $\begin{array}{l}21 \\
(2.3)\end{array}$ \\
\hline $\begin{array}{l}\text { Bad Obstetric } \\
\text { History }(\mathrm{BOH})\end{array}$ & - & - & - & - & - & $\begin{array}{l}10 \\
(5.5)\end{array}$ & $\begin{array}{l}16 \\
(7.3)\end{array}$ & $\begin{array}{l}6 \\
(2.6)\end{array}$ & $\begin{array}{l}2 \\
(0.7)\end{array}$ & $\begin{array}{l}34 \\
(3.7)\end{array}$ \\
\hline Others & $\begin{array}{l}11 \\
(4.9)\end{array}$ & $\begin{array}{l}10 \\
(4)\end{array}$ & $\begin{array}{l}25 \\
(9.4)\end{array}$ & $\begin{array}{l}25 \\
(8.5)\end{array}$ & $\begin{array}{l}71 \\
(6.9)\end{array}$ & $\begin{array}{l}7 \\
(3.8)\end{array}$ & $\begin{array}{l}5 \\
(2.3)\end{array}$ & $\begin{array}{l}10 \\
(4.3)\end{array}$ & $\begin{array}{l}7 \\
(2.4)\end{array}$ & $\begin{array}{l}29 \\
(3.1)\end{array}$ \\
\hline
\end{tabular}

* Chi square $=9.782, \mathrm{p}=0.001 ;{ }^{\#}$ Chi square $=9.956, \mathrm{p}=0.001 ;{ }^{\$}$ Chi square $=3.159 ; \mathrm{p}=0.07$

The leading indication for CS in the present study was fetal distress overall $(34.9 \%)$ as well as for each of the four years. The rate did not vary significantly over the four years. This was followed by previous CS (25.9\%), CPD (10.4\%), malpresentation/malposition (8.2\%) and prolonged labour (7.8\%). The ICMR study by Kambo et al at reported on data from 30 teaching institutions in the years 1998-1999 and found the most frequent indication to be dystocia $(37.5 \%)$ followed by fetal distress with or without meconium aspiration (33.4\%), repeat CS (29\%) and malpresentation (14.5\%). ${ }^{13}$ The ICMR task force study from the same 30 institutions carried out in the year 2005-2006 reported the most frequent indication for primary CS to be CPD (52.9\%), fetal distress (25.8\%), severe PIH/eclampsia $(5 \%) .{ }^{19}$

Unnikrishnan et al from Mangalore found 'previous caesarean delivery' to be most frequent (32.7\%) indication for CS followed by fetal distress (19.6\%) and Breech presentation (10.3\%). ${ }^{5}$ Parity-wise analysis done 
by them revealed the common indications amongst the primigravidae to be fetal distress $(31.1 \%)$ followed by Breech (15.2\%), failed induction (14.3\%) and severe PIH $(8.9 \%) .^{5}$ Among the multigravidae, they found the most frequent indication to be previous CS $(50 \%)$ followed by fetal distress (17\%), breech (9\%), severe PIH (7\%) and failed induction $(6 \%){ }^{5}$ In the present study as well, the commonest indication amongst the primigravidae was fetal distress $(47.5 \%)$ and the commonest indication amongst the multigravidae was previous CS (54.8\%) followed by fetal distress $(20.8 \%)$. Further, among the multigravidae who had no prior CS delivery, fetal distress $(52.1 \%)$ was the leading indication followed by malposition/malpresentation (14\%) and prolonged labour $(8.1 \%)$. An audit from the United Kingdom reported the top five indications to be fetal compromise (22\%) followed by "failure to progress" $(20 \%)$, repeat CS (14\%), breech $(11 \%)$ and "maternal request" $(7 \%){ }^{22}$ The last mentioned indication has been on a rise globally and in India as well and is ethically questionable.

Table 4: Indications for caesarean section among multigravidae with previous vaginal delivery over four years (2008-2011; $\mathrm{N}=420)$.

\begin{tabular}{|c|c|c|c|c|c|}
\hline $\begin{array}{c}\text { Indication for Caesarean } \\
\text { Section }\end{array}$ & $\begin{array}{c}2008 \\
n=87 \\
(\%)\end{array}$ & $\begin{array}{c}2009 \\
n=119 \\
(\%)\end{array}$ & $\begin{array}{c}2010 \\
n=98 \\
(\%)\end{array}$ & $\begin{array}{c}2011 \\
n=116 \\
(\%)\end{array}$ & $\begin{array}{l}\text { All four years } \\
\begin{array}{c}\text { (2008-2011) } \\
\text { N=420 } \\
(\%)\end{array}\end{array}$ \\
\hline Fetal Distress & $\begin{array}{c}41 \\
(47.1)\end{array}$ & $\begin{array}{c}68 \\
(57.1)\end{array}$ & $\begin{array}{c}55 \\
(56.1)\end{array}$ & $\begin{array}{c}55 \\
(56.1)\end{array}$ & $\begin{array}{c}219 \\
(52.1)\end{array}$ \\
\hline $\begin{array}{l}\text { Malposition/ } \\
\text { Malpresentation }\end{array}$ & $\begin{array}{c}14 \\
(16.1)\end{array}$ & $\begin{array}{c}18 \\
(15.1)\end{array}$ & $\begin{array}{c}15 \\
(15.3)\end{array}$ & $\begin{array}{c}12 \\
(10.3)\end{array}$ & $\begin{array}{c}59 \\
(14)\end{array}$ \\
\hline Prolonged labour & $\begin{array}{c}11 \\
(12.6)\end{array}$ & $\begin{array}{c}6 \\
(5)\end{array}$ & $\begin{array}{c}4 \\
(4.1)\end{array}$ & $\begin{array}{c}13 \\
(11.2)\end{array}$ & $\begin{array}{c}34 \\
(8.1)\end{array}$ \\
\hline $\begin{array}{l}\text { Cephalo-Pelvic } \\
\text { Disproportion (CPD) }\end{array}$ & $\begin{array}{c}3 \\
(3.4) \\
\end{array}$ & $\begin{array}{c}7 \\
(5.9) \\
\end{array}$ & $\begin{array}{c}6 \\
(6.1) \\
\end{array}$ & $\begin{array}{c}7 \\
(6) \\
\end{array}$ & $\begin{array}{c}23 \\
(5.5 \%)\end{array}$ \\
\hline $\begin{array}{l}\text { Hypertensive Disorders of } \\
\text { Pregnancy (HDP) }\end{array}$ & $\begin{array}{c}1 \\
(1.1)\end{array}$ & $\begin{array}{c}1 \\
(0.8)\end{array}$ & $\begin{array}{c}5 \\
(5.1)\end{array}$ & $\begin{array}{c}10 \\
(8.6)\end{array}$ & $\begin{array}{l}17 \\
(4)\end{array}$ \\
\hline $\begin{array}{l}\text { Antepartum Haemorrhage } \\
(\mathrm{APH})\end{array}$ & $\begin{array}{c}5 \\
(5.7)\end{array}$ & $\begin{array}{c}7 \\
(5.9)\end{array}$ & $\begin{array}{c}3 \\
(3.1)\end{array}$ & $\begin{array}{c}6 \\
(5.2)\end{array}$ & $\begin{array}{l}21 \\
(5)\end{array}$ \\
\hline $\begin{array}{l}\text { Bad Obstetric History } \\
(\mathrm{BOH})\end{array}$ & $\begin{array}{c}2 \\
(2.3) \\
\end{array}$ & $\begin{array}{c}5 \\
(4.2) \\
\end{array}$ & $\begin{array}{c}4 \\
(4.1 \%) \\
\end{array}$ & $\begin{array}{c}1 \\
(0.9)\end{array}$ & $\begin{array}{c}12 \\
(2.9)\end{array}$ \\
\hline Obstructed Labour & $\begin{array}{c}1 \\
(1.1)\end{array}$ & $\begin{array}{c}2 \\
(1.7) \\
\end{array}$ & $\begin{array}{c}2 \\
(2) \\
\end{array}$ & $\begin{array}{c}4 \\
(3.4)\end{array}$ & $\begin{array}{c}9 \\
(2.1) \\
\end{array}$ \\
\hline Others & $\begin{array}{c}9 \\
(10.3)\end{array}$ & $\begin{array}{c}5 \\
(4.2) \\
\end{array}$ & $\begin{array}{c}4 \\
(4.1) \\
\end{array}$ & $\begin{array}{c}8 \\
(6.9) \\
\end{array}$ & $\begin{array}{c}26 \\
(6.2)\end{array}$ \\
\hline
\end{tabular}

A study from Pakistan in 2014 reports the CS rate to be $45.5 \%$ at a military hospital with the commonest indication being repeat CS $(44.4 \%)$ followed by failed trial of labour $(27.1 \%)$ and fetal distress $(10.1 \%) .{ }^{11}$ An audit of CS in Srilanka carried out in 2010 reported previous $\mathrm{CS}$ as most frequent $(35.6 \%)$ indication followed by fetal distress $(22.3 \%)$ and failure to progress $(10.8 \%){ }^{16}$ They recommended use of Robson's 10 group classification of CS for auding $\mathrm{CS} .{ }^{16}$ The same has also been recommended by the WHO in a recent release $2015^{8}$

From the above discussion and findings it emerges that fetal distress and repeat CS are two major indications that should be explored for reducing the CS rates. The definition of fetal distress/compromise for which CS delivery should be offered must be unambiguous. It is well known that use of Cardiotocography (CTG) leads to over diagnosis of fetal distress. The accurate method of diagnosing fetal distress is fetal scalp $\mathrm{pH}$ but its availability is limited in the developing world. On the other hand, CTG being non-invasive and being more readily available over time is contributing to the increasing figures of fetal distress. Hence a comprehensive assessment by one or more physicians may be in order before deciding to deliver a primigravida by CS.

The way to reducing CS for previous CS lies in reducing the primary CS per se, and also in judiciously considering Vaginal Birth after CS (VBAC) in deserving patients. The success rate of VBAC reported by the ICMR task force study from teaching institutions in the year 20052006 was $62.3 \% .^{19}$

Caesarean section rate at a population level is an indicator of accessibility, availability and utilization of this facility, and is of use to policymakers as an indicator 
of maternal/perinatal health. ${ }^{1,8}$ At hospital level however, the rates need to be monitored and the WHO recommends the Robson's ten group classification system for the same. ${ }^{8}$ The ICMR study by Kambo et al noted maternal and perinatal mortality to be 299/100000 and 493/1000 deliveries respectively, which is high inspite of increasing CS rate $^{13}$ The recent publication from WHO also highlights the need for studying maternal and perinatal morbidity indicators (short term and long term such as birth asphyxia, obstetric fistulae, etc.) in relation to CS rates for optimizing the outcomes than just mortality statistics. ${ }^{8}$

\section{CONCLUSIONS}

There is a definite need to continuously monitor the CS rates in the institutions and the WHO recommended ten group classification may be adopted for the same. The caesareans done for fetal distress and previous CS need to be particularly audited as these are the major contributors to rising CS rates. There is a need for deciding and defining at the institute level, the measures of 'fetal distress' for which CS delivery should be offered. Careful assessment of parous women with previous CS to select the candidates for VBAC may also reduce the repeat CS rate. It is of crucial importance to make a comprehensive and judicious assessment prior to deciding the primary CS.

Funding: No funding sources

Conflict of interest: None declared

Ethical approval: The study was approved by the Institutional Ethics Committee

\section{REFERENCES}

1. Betran AP, Merialdi M, Lauer JA, Bing-shun W, Thomas J, van Look P, et al. Rates of caesarean section: Analysis of global, regional and national estimates. Pediatric and Perinatal Epidemiology. 2007;21:98-113.

2. Roy Choudhary C. Caesarean births: the Indian scenario. Abstract. Presented at the Population Association of America 2008 Annual meeting. 17-19 April 2008. New Orleans, USA.

3. Stanton CK, Holtz SA. Levels and trends in caesarean births in the developing world. Studies in Family planning. 2006;37(1):41-8.

4. Ado D, Geidam BM, Audu BM, Kawuwa J, Obed Y. Rising trends and indications of caesarean section at the university of Maiduguri teaching hospital Nigeria. Annals of African Medicine. 2009;8(2):12732.

5. Unnikrishnan B, Rakshith P, Aishwarya A, Nithin K, Rekha T, Prasanna P, et al. Trends and Indications for Caesarean Section in a tertiary care Obstetric Hospital in Coastal South India. Australasian Medical Journal. 2010;3:821-5.
6. WHO Joint interregional conference on appropriate technology for birth. Fortaleza, Brazil, 22-26 April, 1985.

7. World Health Organization. Appropriate technology for birth revisited. $\mathrm{Br} \mathrm{J}$ Obstet Gynaecol. 1992;99:709-10.

8. Department of Reproductive Health and Research. WHO statement on Caesarean Section rates, 2015. World Health Organization, Geneva.

9. Mukherjee SN. Rising caesarean section rate. J Obstet Gynecol India. 2006;56(4):298-300.

10. Naidoo N, Moodley J. Rising rates of caesarean sections in specialist private practice. SA Fam Pract. 2009;51(3):254-8.

11. Sajjad R, Ali CA, Iqbal A, Sajjad N, Haq MZ. An audit of cesarean sections in Military Hospital Rawalpindi. Anaesth Pain \& Intensive Care 2014;18(2):172-5

12. Goonewardene M, Kumara DMA, Arachchi DRJ, Vithanage R, Wijeweera $\mathrm{R}$. The rising trend of caesarean section rates: should we and can we reduce it? Sri Lanka Journal of Obstetrics and Gynaecology. 2012;34:11-8.

13. Kambo I, Bedi N, Dillon BS, Saxena NC. A critical appraisal of caesarean sectionrates at teaching hospital in India. International J Obstet Gynaecol. 2002;79:151-8.

14. Ghosh S. Increasing trends in caesarean section delivery in India, Role of medicalization of maternal health. In Working Papers. The Institute of Social and Economic Change, Banglore, 2010.

15. Gibbons L, Belizan JM, Lauer JA, Betran AP, Merialdi M, Althabe F. The global numbers and costs of additionally needed and unnecessary caesarean sections performed per year: Overuse as a barrier to universal coverage. World Health Report, 2010.

16. Goonewardene M, Manawadu MH, Priyaranjana DV. Audit: The strategy to reduce the rising caesarean section rates. J South Asain Feder Obstet Gynae. 2012;4(1):5-9.

17. Pahari K, Ghosh A. Study of pregnancy outcome over a period of five years in a postgraduate Institute of West Bengal. J Ind Med Assoc. 1997;95(6):172-4.

18. Sreevidya S, Sathiyasekaran BW. High caesarean rates in Madras (India): a population-based cross sectional study. $\mathrm{Br}$ J Obstet Gynaecol. 2003;110(2):106-11.

19. Dhillon BS, Chandhiok N, Bhatia BS, Coyaji KJ, Das MC, Das V, et al. Vaginal birth after caesarean section (VBAC) versus emergency caesarean section at teaching hospitals in India: an ICMR task force study. Int J Reprod Contracept Obstet Gynecol. 2014;3(3):592-7.

20. Dhodapkar SB, Bharavi S, Daniel M, Chauhan NS, Chauhan RC. Analysis of caesarean sections according to Robson's ten group classification system at a tertiary care teaching hospital in South India. Int $\mathbf{J}$ Reprod Contracept Obstet Gynecol. 2015;4(3):745-9. 
21. Balakrishnan D, Nair VR. Our exploding cesarean rates: A system for auditing. Journal of Evolution of Medical and Dental Sciences. 2014;3(14):3563-7.

22. Thomas J, Paranjothy S. Royal College of Obstetricians and Gynaecologists, Clinical Effectiveness Support Unit. The National Sentinel
Caesarean Section Audit Report. London: RCOG Press, 2001.

Cite this article as: Bharadwaj M, Modi J. A four year audit of deliveries by Caeserean section at a medical college hospital in Central India. Int $\mathbf{J}$ Reprod Contracept Obstet Gynecol 2015;4:1775-82. 